\title{
Recovering facular areas through Doppler imaging
}

\author{
V. Aarum-Ulvås ${ }^{1,2,3}$ \\ 1 The Research Council of Norway, PO Box 2700 St. Hanshaugen, 0131 Oslo, Norway \\ 2 Astrophysikalisches Institut Potsdam, An der Sternwarte 16, 14482 Potsdam, Germany \\ e-mail: vaarum@aip.de \\ ${ }^{3}$ Institute of Theoretical Astrophysics, PO Box 1029 Blindern, 0315 Oslo, Norway
}

Received 7 June 2004 / Accepted 1 February 2005

\begin{abstract}
Similar to the Sun, stellar active regions appear to consist of dark spots in combination with surrounding bright facular regions. The present study uses the Doppler imaging technique to recover the stellar surface structures from simulated spectral observations obtained from a given theoretical stellar surface. The objective of the study is to investigate how the recovered surface structure depends on observing parameters such as rotational phase coverage, choice of spectral lines, signal-to-noise ratio, and projected equatorial rotational velocity. The inversions demonstrate that those stellar surface active regions with high temperature contrast in both longitudinal and latitudinal directions can be satisfactorily reproduced using Doppler imaging. Observations must, however, fulfill such strict conditions that a complete reconstruction is somewhat unrealistic. Nevertheless, in a more realistic example, the hot facular areas result in quite distinct and easily recognizable features on the stellar surface map, even though they are not completely reconstructed.
\end{abstract}

Key words. methods: data analysis - techniques: spectroscopic - stars: activity - stars: atmospheres - stars: imaging stars: starspots

\section{Introduction}

Even the smallest sunspots are always accompanied by a brightening of the surrounding atmosphere, known as facular areas (see e.g. Rast et al. 2001; Foukal 2004). The faculae are easily seen in white light near the solar limb and in monochromatic spectral line radiation across the solar disc. Variations in total solar irradiance caused by the active regions (spots and faculae combined) are about $0.1 \%$ (Krivova et al. 2003, and references therein). A ground-based telescope taking photometric observations of late-type stars would not detect such small variations. However, one would expect the effect of the facular regions to be stronger for stars with higher activity levels than the Sun's.

Studies of the spatial correlation have been carried out between dark spots on stellar surfaces, on the one hand, and facular regions, chromospheric plage, and transition-region emission of the same stars, on the other. Some of these studies indicate that the emission regions in some cases are concentrated near, or even surrounding, starspots (see e.g. Donati et al. 1992; Saar et al. 1992; Strassmeier et al. 1993; Strassmeier 1994; Taş et al. 1999; Lanza et al. 2001).

A similar study of the triple-lined RS CVn-type system UX Ari (HD 21242) was published by Huenemoerder et al. (1989), who found that the chromospheric emission of UX Ari was rotationally modulated and that maximum emission occurred slightly earlier in rotational phase than the photometric minimum. This suggests that the chromospheric emission originates close to photospheric spots.

Photometric observations of UX Ari have also revealed a distinct relation between $V$ magnitude and $B-V$ colour index, for the UX Ari system as a whole gets bluer as it gets darker (Aarum Ulvås \& Henry 2003, and references therein). Aarum Ulvås \& Engvold (2003) demonstrated that this colourbrightness relation cannot be explained by how the bluer flux of the hotter secondary component contributes more to the total system flux when the cool starspots of the primary component come into view. It is necessary to introduce bright facular regions on the surface of the primary component, in order to explain the observed relation between brightness and colour.

There is reason, therefore, to believe that dark spots and bright facular regions can exist close to each other on the surfaces of active stars. However, there is also reason for concern about whether Doppler imaging programs are able to reconstruct such features, particularly if the dark and bright regions lie close to each other along lines of the same projected rotational velocity. Surface areas with different temperatures that lie along the same longitude and close to each other in latitude are difficult to separate using spectroscopic observations, since the spectral features caused by the hot and cool areas enter and leave the spectral line profiles almost simultaneously. A dark spot with bright regions just north and/or south of it is easily interpreted by the Doppler imaging program as a dark spot 
with size corresponding to the total size of the dark and bright areas and with temperature that is slightly higher than the true temperature of the dark spot.

To test whether the Doppler imaging technique is able to reconstruct surface features like the one described above, inversions of synthetic spectroscopic data were performed. The present paper describes how these inversions were done and discusses their implications for the Doppler imaging technique.

\section{Simulations of starspots and faculae}

Inversions described in this paper used the INVERS7-9PD spectral line profile inversion code originally developed by N. Piskunov and later modified by T. Hackman (Piskunov et al. 1990; Piskunov 1991; Hackman et al. 2001). All spectral line profiles that were inverted were synthetically generated as described in the following.

\subsection{Generating synthetic data}

The stellar surface used in generating the synthetic observations is shown in Fig. 1. The surface has an active region consisting of a cool dark spot surrounded by a bright facular ring to represent a surface feature with high temperature gradients along both latitude and longitude. The surface effective temperature in Fig. 1 is $4750 \mathrm{~K}$ in the photosphere, $3750 \mathrm{~K}$ in the spot, and $5250 \mathrm{~K}$ in the ring. This photospheric temperature is appropriate for UX Ari (Vogt \& Hatzes 1991).

The facular ring is modelled here by a simple temperature increase in the photosphere. This is not in accordance with solar facular models, where the temperature contrast depends on the limb angle and width of the magnetic flux tubes forming the active region. However, those stars that are targets for Doppler imaging display activity several orders of magnitude stronger than the Sun. It is appropriate to expect their faculae to appear bright also against the centre of the stellar disc. The simple temperature increase used here is almost certainly too simple a model, but it is as good as any until our knowledge of stellar faculae increases.

Synthetic high resolution spectroscopic data $(\lambda / \Delta \lambda=$ $80000)$ equidistant in rotational phase $\varphi$ were generated using a surface grid of $3^{\circ}$ in both longitude and latitude. Other parameters affecting the synthetic line profiles are given in Table 1. Inclination $i$ of the rotational axis was not the same for all the inversions. See Sect. 3.2 for details. The generated data were convolved with an instrumental profile, and artificial noise was added with a given signal-to-noise ratio.

Synthetic $V, B-V$, and $U-B$ photometric observations could also be generated from this star, which were then compared to the $V, B-V$, and $U-B$ photometry that was deduced from the temperature distribution that was output from the inversion program. The synthetic photometric observations were not used by INVERS7-9PD as an additional constraint in the surface reconstruction.

Whenever the word "observations" is set in quotation marks in the following, it refers to the synthetic data (spectroscopic or photometric) that were generated as described above.

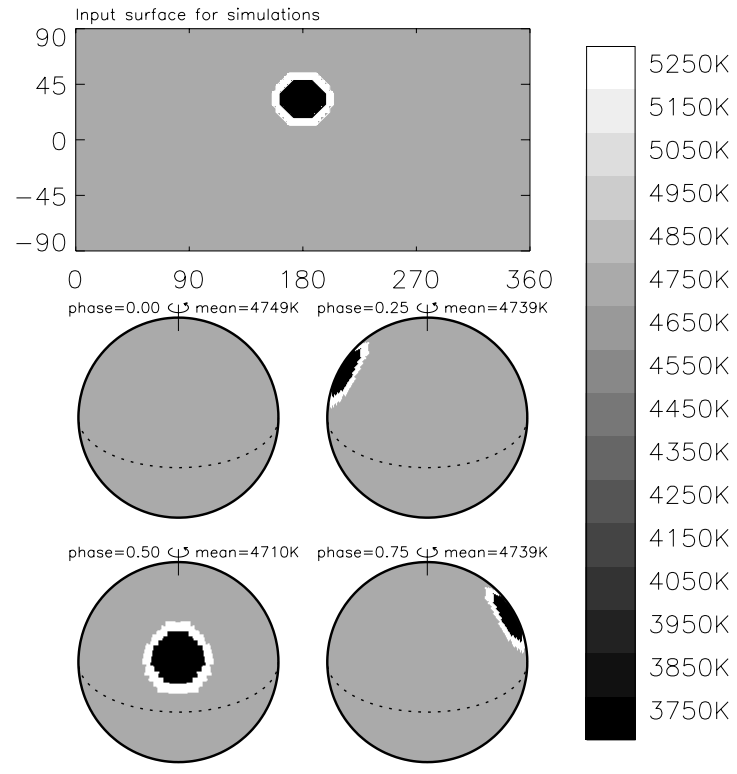

Fig. 1. The input stellar surface temperature distribution. The Mercator projection at the top shows stellar longitude along its abscissa and stellar latitude along its ordinate. Below the Mercator projection are four spherical projections of the stellar surface at four different rotational phases, given above each projection along with the average effective temperature of all the visible surface pixels. Note that the rotational phase and the stellar longitude run in opposite directions. To the right is a bar linking greyscale and effective temperature. The surface effective temperature is $4750 \mathrm{~K}$ in the photosphere, $3750 \mathrm{~K}$ in the spot, and $5250 \mathrm{~K}$ in the ring.

Table 1. Stellar parameters used when generating synthetic data. Inversions with other values of $i$ are described in Sect. 3.2.

\begin{tabular}{ll}
\hline \hline Parameter & Value \\
\hline Surface gravity $\log g\left(\mathrm{~cm} \mathrm{~s}^{-1}\right)$ & 3.0 \\
Chemical element abundances $[\mathrm{Fe} / \mathrm{H}]$ & 0.0 \\
Inclination $i$ & $60^{\circ}$ \\
Microturbulence velocity & $1.7 \mathrm{~km} \mathrm{~s}^{-1}$ \\
Macroturbulence velocity (radial and tangential) & $3.0 \mathrm{~km} \mathrm{~s}^{-1}$ \\
\hline
\end{tabular}

\subsection{Choice of spectral lines}

In order to ease reconstruction of high temperature contrast active regions, especially in the latitudinal direction, it was decided to search for a set of spectral lines that differed in temperature sensitivity, in the sense that some lines were sensitive to temperature change from $4750 \mathrm{~K}$ to $3750 \mathrm{~K}$ (corresponding to the temperature difference between the photosphere and the spot), and some were sensitive to temperature change from $4750 \mathrm{~K}$ to $5250 \mathrm{~K}$ (from photosphere to ring). A combination of spectral lines with different temperature sensitivities was assumed to facilitate the reconstruction further.

The search was performed by generating spectra for the same stellar model atmosphere at different temperatures $(3750 \mathrm{~K}, 4750 \mathrm{~K}$, and $5250 \mathrm{~K})$ and by visual inspection to determine which lines were the most temperature sensitive. The spectra were calculated for a non-rotating star, in order for rotation not to influence the integrated spectral line profiles. 

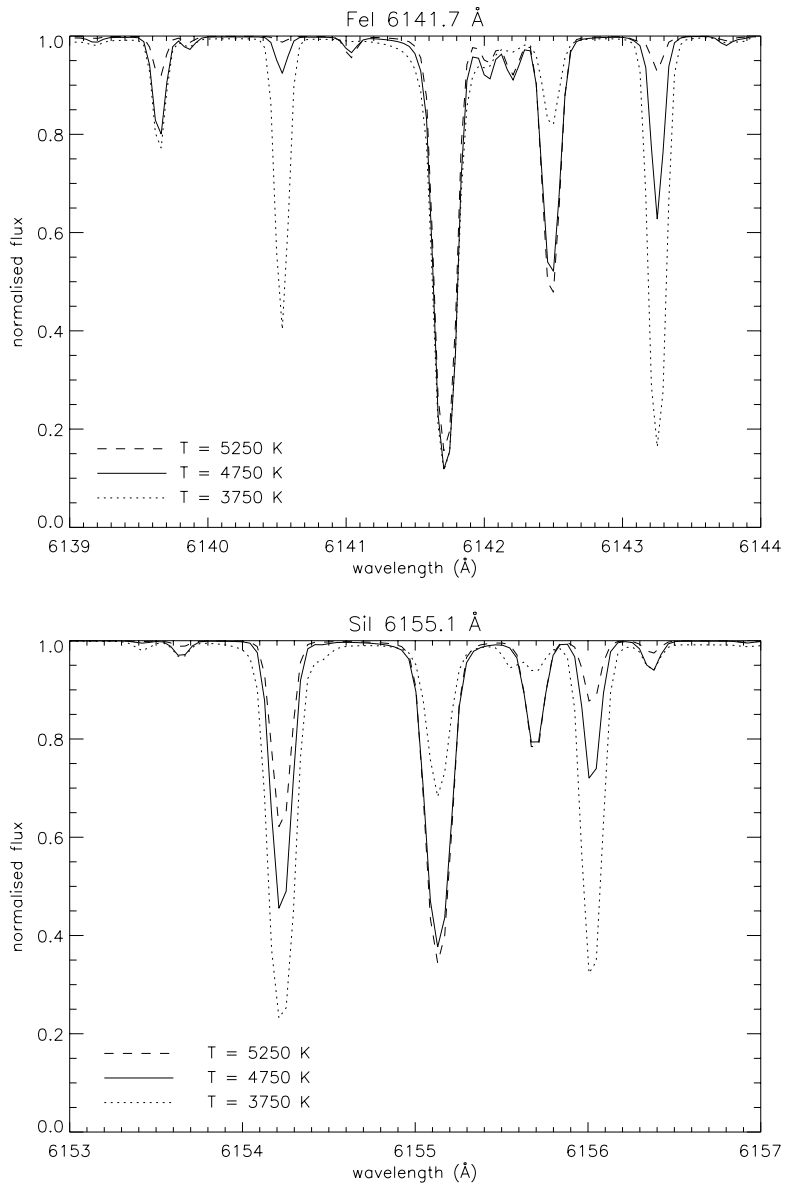

Fig. 2. Spectral regions surrounding the FeI $6141.7 \AA$ and Si I $6155.1 \AA$ lines calculated for three different temperatures. The spectral regions shown correspond roughly to the regions actually used in the inversions. The main spectral lines in each region are identified in the text.

The micro- and macroturbulent velocities were the same as in Table 1.

The most sensitive lines thus selected were subsequently used in inversions of synthetic spectra calculated for a rapidly rotating model star with a surface feature as shown in Fig. 1. Again, visual inspections determined how close to the correct stellar surface the inversion result came for each line, and various combinations of these lines were also tested.

The best results were obtained using a combination of two line complexes, usually referred to in the literature as Fe I $6141.7 \AA$ and SiI $6155.1 \AA$. The two lines and their immediate surroundings are shown in Fig. 2, calculated for the three different temperatures from Fig. 1. The spectral regions in Fig. 2 correspond closely to the regions that are actually used when inverting the spectra of rapidly rotating stars. Both regions consist of several spectral lines with different temperature sensitivities. The four strongest lines surrounding FeI $6141.7 \AA$ are $\mathrm{Fe}_{\mathrm{I}} 6139.6 \AA$, ZrI $6140.5 \AA$, Si I $6142.5 \AA$, and Zr I $6143.3 \AA$. The three strongest lines surrounding Si I $6155.1 \AA$ are Na I $6154.2 \AA$, Si I $6155.7 \AA$, and Ca I 6156.0 A.

In order to produce the spectral line profiles that would be observed from a rapidly rotating star, spectral contributions
Table 2. Spectral lines that display similar behaviour to Fe I $6141.7 \AA$ and Si I $6155.1 \AA$ in the temperature range from $3750 \mathrm{~K}$ to $5250 \mathrm{~K}$ and that have previously been used for Doppler imaging.

\begin{tabular}{ccc}
\hline \hline Ti I 5866.4 & Fe I 6180.2 & Li I 6707.9 \\
Ca I 6102.8 & VI 6199.2 & Ca I 6717.7 \\
Ni I 6108. $\AA$ & Fe I 6430.9 & Ti I 6743.1 \\
Ca I 6122.2 & Ca I 6439.1 & Ca I 7148.2 \\
Fe I 6151.6 & Fe I 6546.2 & KI 7699.0 \\
Ca I 6166.4 & Ni I 6643.6 & Ti I 8412.4 \\
Fe I 6173.3 & & \\
\hline
\end{tabular}

(like the ones in Fig. 2) from each point on the visible stellar disc are integrated. Each contribution is shifted in wavelength according to the local rotational velocity, and the result for each of the spectral regions in Fig. 2 is one rotationally broadened line profile. Since the different spectral lines making up the rotationally broadened profiles have different temperature sensitivities, different parts of the disc-integrated profiles respond differently to a local change in temperature on the stellar surface. The varying temperature sensitivity across the observed line profiles increases the ability to reconstruct both hot and cool areas on the stellar surface.

Which spectral line combination is the most favourable to use will depend on the temperatures in the photosphere, spots, and faculae. It will therefore vary from one target star to another. Table 2 shows other spectral lines that display similar behaviour as the lines used in this study and that have previously been used for Doppler imaging.

\section{Results and discussion}

\subsection{Optimal parameter values}

Using the proper set of spectral lines from Sect. 2.2, synthetic spectroscopic observations generated from the star shown in Fig. 1 were inverted. The parameters of the synthetic observations and the inversion procedure itself were varied in the search for a satisfactory reconstruction of the stellar surface active region. It was found that the following parameter values gave a satisfactory result:

- $S / N=400$ in the synthetic spectra.

- 40 spectral "observations" equidistant in rotational phase $(0.000,0.025,0.050, \ldots, 0.975)$.

- $v \sin i=80 \mathrm{~km} \mathrm{~s}^{-1}$ for the target star.

- 40 iterations in the inversions.

The values of $i$ and $v \sin i$ in the inversion were fixed to values from the generation of synthetic data. When inverting real observations of a star, $v \sin i$ would be derived from the observed rotationally broadened line profiles, and $i$ could be roughly estimated from the radial velocity curve if the observed star is a spectroscopic binary. The effects of using incorrect values of $i\left(\Delta i= \pm 10^{\circ}\right.$ and $\left.\pm 20^{\circ}\right)$ and $v \sin i\left(\Delta v \sin i= \pm 1 \mathrm{~km} \mathrm{~s}^{-1}\right.$, 

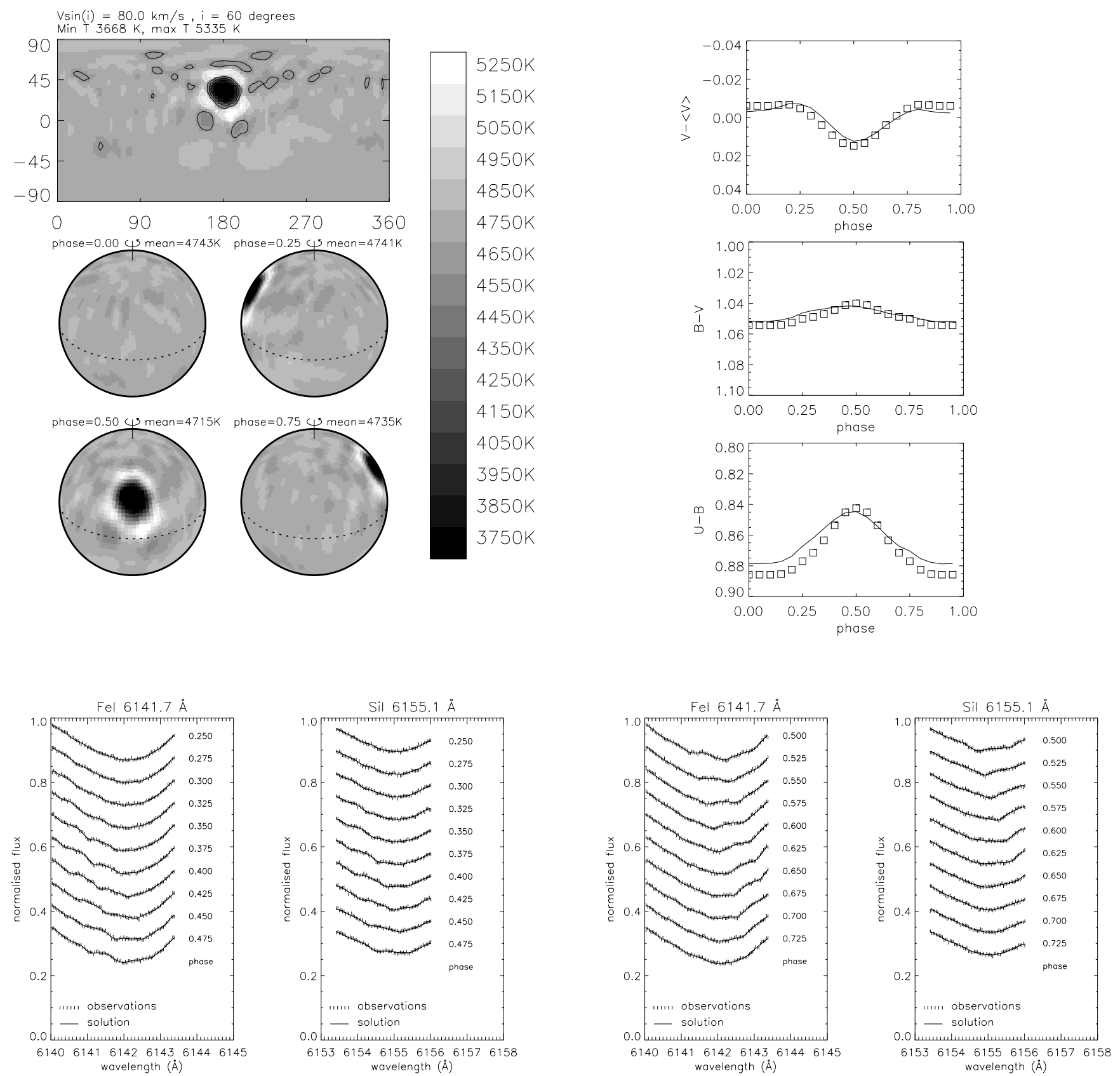

Fig. 3. Reconstructing the stellar surface consisting of a dark spot surrounded by a bright ring. Top left: the reconstructed stellar surface. The projections, annotations and temperature scale are as in Fig. 1, with some additional annotations on top of the Mercator projection. These give the projected equatorial rotational velocity $v \sin i$, the inclination $i$, and the minimum and maximum effective temperatures in the surface pixels. Top right: the "observed" (squares) and reconstructed (solid line) photometry (from top to bottom: the $V$ band light curve, the $B-V$ colour curve, and the $U-B$ colour curve). Bottom: the "observed" (tick marks) and reconstructed (solid lines) spectral line profiles in the phase interval $0.250-0.725$.

$\pm 2 \mathrm{~km} \mathrm{~s}^{-1}, \pm 3 \mathrm{~km} \mathrm{~s}^{-1}$, and $\pm 4 \mathrm{~km} \mathrm{~s}^{-1}$ ) in the inversion were investigated, and the results were similar to those described by Vogt et al. (1987). Already a deviation of $\pm 1 \mathrm{~km} \mathrm{~s}^{-1}$ in $v \sin i$ caused several dark and bright bands to appear parallel to the equator. As also noticed by Vogt et al., Doppler imaging is so sensitive to errors in this parameter that its true value can actually be determined by choosing the value that produces the least systematic variation of surface brightness with latitude. Doppler imaging is less sensitive to errors in $i$, but also using the correct value here resulted in the least systematic brightness variation and the best agreement between the model and "observations".

The result from the optimal inversion is shown in Fig. 3. The inversions started with an initial guess of a smooth stellar surface. The ring is completely recovered, although there is a small gap between the spot and the ring on the equator side of the spot. This, and the fact that the ring is slightly wider towards the equator, illustrates the reduced latitudinal resolution 
in the equatorial areas of stars with high inclination, which is a general weakness of the Doppler imaging technique (Vogt et al. 1987; Rice et al. 1989).

The spectral line profiles shown in Fig. 3 cover only the rotational phase interval from 0.250 to 0.725 . These are the phases influenced by the active region centred on phase 0.500 . The mean deviation between the "observed" spectrum and the model spectrum is $0.243 \%$, corresponding to $S / N=412$ in the synthetic observations. The reconstructed spot temperature $(\approx 3770 \mathrm{~K})$ is only $\approx 20 \mathrm{~K}$ higher than the input spot temperature, which can be characterized as a successful reconstruction. The reconstructed facular temperature $(\approx 5170 \mathrm{~K})$ is $\approx 80 \mathrm{~K}$ lower than the input facular temperature. The same inversion, but with the facular ring as the only active region, revealed surprisingly that the ring is more easily reconstructed when the spot is in place. A possible reason might be that the ring set at $500 \mathrm{~K}$ warmer than the photosphere has a smaller effect on the stellar spectrum than the spot set at $1000 \mathrm{~K}$ cooler than the photosphere.

The "observed" and the reconstructed $V$ magnitudes in Fig. 3 coincide satisfactorily. The $V$ amplitude is quite small due to the presence of facular areas close to the spot. The reconstructed $B-V$ colour shows the same general behaviour as the "observed" colour; i.e. it becomes slightly bluer at the phases where the spot and ring are visible. This suggests that the effect of the hot ring on the $B-V$ colour is greater than the effect of the spot. The reconstructed colour is less symmetrical with phase than the "observations". The result for the $U-B$ colour is basically the same as for the $B-V$ colour, but the amplitude is larger, which demonstrates that the hot ring is more easily visible in bluer wavelengths than in redder ones.

This inversion demonstrates that it is possible to use Doppler imaging to reconstruct a stellar surface active region consisting of a cool, dark spot surrounded by a hot, bright ring. However, a successful reconstruction requires spectroscopic observations that are hard to achieve in practice, particularly concerning the signal-to-noise ratio and rotational phase coverage.

\subsection{Results from less optimal parameter values}

We have seen (Fig. 3) that it is possible to reconstruct high temperature contrast active regions on the surfaces of stars through the use of Doppler imaging. The spectroscopic observations used as the Doppler imaging input must, however, fulfill several requirements that may seem unrealistic, particularly as far as the signal-to-noise ratio and the rotational phase coverage are concerned. The following is an investigation of the effects of varying some parameters of the "observations", the star, and the activity region. The following cases are considered:

- Lower signal-to-noise ratio in the "observations".

- Poorer rotational phase coverage.

- Lower rotational velocity.

- Active region located at different latitudes.

- Varied size of the active region.

- Lower temperature contrast between the spot and the ring.

- Varied stellar inclination.
- Introduction of a polar spot in addition to the active region from Fig. 3.

- Several active regions like the one in Fig. 3 present on the stellar surface at the same time.

Each of these cases is considered in turn.

\subsubsection{Signal-to-noise ratio}

In the successful reconstruction of the active region in Fig. 3 the synthetic spectroscopic observations had a signal-to-noise ratio $S / N=400$. Here we investigate how the reconstructed stellar surface is affected if $S / N=200$.

As long as the observations have high quality - i.e. they have high $S / N$, high spectral resolution, dense phase coverage, are free from line blends, and data reduction errors, etc. - it does not matter much what method of regularization is used to constrain the solution. The outcome of Doppler imaging in this case is well constrained by the data alone. Only when $S / N$ becomes lower does it become more important which regularization method is used.

Two of the most commonly used regularization functions are the maximum entropy method (MEM, Vogt et al. 1987) and Tikhonov regularization (Piskunov et al. 1990). Both methods prefer a smooth output map, but use different approaches to obtain it. Where MEM seeks to maximize the entropy of the map, Tikhonov regularization seeks to minimize the gradients. This implies that Tikhonov regularization assumes a certain degree of correlation between neighbouring pixels in the map, something MEM does not.

When it comes to the case at hand, tests by Vogt et al. (1987) have shown that MEM tends to underestimate the spot-to-photosphere contrast when observations are noisy. This would correspond to too high a spot temperature and too low a facular temperature. Tikhonov regularization can be regarded as a low-pass filtering in spatial frequency (Piskunov et al. 1990). Stronger noise in the observations means that the highest spatial frequency allowed in the map is reduced, and the map becomes smoother, which in turn would mean blurring the borders between the spot and ring and between the ring and photosphere. The test performed here shows that this is indeed what happens, as there is a narrow gap of photospheric temperature between the spot and the ring. The ring also seems to be wider than on the original surface, although it is still recovered. The stellar surface outside the active region becomes less smooth as a consequence of the increased noise level in the "observations".

An increased noise level in the observations affects the obtainable resolution in the resulting map, but the broad features of the stellar surface are still recovered.

\subsubsection{Rotational phase coverage}

40 spectroscopic observations equidistant in rotational phase $\varphi$ $(0.000,0.025, \ldots, 0.975)$ were required for a satisfactory reconstruction of the bright facular ring (Fig. 3). An inversion was made from "observations" representing 20 equidistant phases $(0.00,0.05, \ldots, 0.95)$. In this case the ring is not 
completely recovered, but instead broken up between the spot and the equator. There is also a gap between the spot and the ring on the equator side of the spot, and the ring is incorrectly extended towards the equator. From this, one concludes that the effect of poorer phase coverage on the latitudinal resolution is greatest near the stellar equator.

\subsubsection{Projected equatorial rotational velocity}

For the inversion shown in Fig. 3, the star had a projected equatorial rotational velocity $v \sin i=80 \mathrm{~km} \mathrm{~s}^{-1}$, while an inversion was also made for $v \sin i=40 \mathrm{~km} \mathrm{~s}^{-1}$. In this case the spot is still recovered, although its shape turns out slightly more square. The ring, on the other hand, is not completely recovered, but is broken up into four parts: directly north, south, east, and west of the spot. The northern part of the ring is correctly positioned and has the correct size. The eastern and western parts of the ring are correctly positioned, but they are incorrectly extended towards the equator. The southern part of the ring is positioned between the spot and the equator, leaving a gap between the spot and the ring.

For a constant spectral resolving power $R$, it appears that lowering the projected equatorial rotational velocity has a significant effect on the surface resolution in the resulting map, especially the latitudinal resolution near the equator. A satisfactory reconstruction would not be possible for increased spectral resolution, i.e. narrower instrumental profile, since the instrumental profile $\left(3.9 \mathrm{~km} \mathrm{~s}^{-1}\right)$ is already narrower than the average local line profile $\left(6.5 \mathrm{~km} \mathrm{~s}^{-1}\right)$. For the set-up used in this test, the longitudinal resolution at the sub-observer latitude was determined by the width of the local line profile.

\subsubsection{Varying the latitude of the active region}

In the successful reconstruction of the active region in Fig. 3 the active region was centred on latitude $30^{\circ}$, which makes it cross the centre of the visible stellar disc. Its line profile distortion correspondingly moves across the whole line profile from the extreme blue wing to the extreme red wing, in exactly half a complete rotation. This latitude is, in other words, the most favourable for reconstruction. In order to investigate the effect of less favourable latitudes, the same active region was centred on the equator and on latitude $60^{\circ}$ in two separate inversion runs.

When the active region is centred on the equator, its line profile distortion covers a shorter distance across the line profile and is visible during less than half a complete rotation. The spot is in this case recovered, but it is centred a little too far to the north. The ring is only partly recovered, i.e. to the east and to the north-west of the spot.

When the active region is centred on latitude $60^{\circ}$, the part of it that lies north of $60^{\circ}$ is always visible, and the line profiles will show it causes some distortion at all phases. Both the spot and the ring are recovered in this case, but the reconstructed ring temperature is a little lower than in the input.

\subsubsection{Varying the size of the active region}

To investigate the effect of different size of the active region, three inversions were performed where the active region had $50 \%, 75 \%$ and $150 \%$, respectively, of the angular size shown in Fig. 1. When the active region had $50 \%$ of its angular size, the ring also had $50 \%$ of its width compared to Fig. 1. The spot is in this case recovered, but the ring is not, because the ring in this case is narrower than one resolution element on the stellar disc (see Sect. 3.2.3). When the whole active region had $75 \%$ of its angular size, the ring had $100 \%$ of its original width. The ring is in this case only partly recovered, i.e. only the parts north and south of the spot. The part of the ring that lies south of the spot is wider than in the input and incorrectly shifted towards the equator.

However, when the active region had $150 \%$ of its angular size, the ring also had $150 \%$ of its original width. The part of the active region that lies closest to the equator is in this case not recovered, neither the spot nor the ring. The spot is cut along a diagonal line crossing its southern half from the southeast towards the north-west, and the ring surrounding this part of the spot, is also not recovered. Instead, a large area with a temperature between the true spot and photosphere temperatures is bounded to the north by the above-mentioned diagonal and to the east and west by the longitudes of the original active region and reaches about $10^{\circ}$ south of the equator. South of the large area is a smaller area with ring temperature. This is again an effect of the reduced latitudinal resolution around the equator.

\subsubsection{Lower temperature contrast between the spot and the ring}

The original spot temperature in Fig. 1 is $1000 \mathrm{~K}$ lower than the photospheric temperature, whereas the facular temperature is $500 \mathrm{~K}$ higher. Faculae with such high temperature are not very difficult to reconstruct with Doppler imaging, but a smaller temperature contrast between the spot and the ring is expected to make the reconstruction of the active region more difficult. This temperature contrast can be decreased by raising the spot temperature, lowering the facular temperature, or both. Tests were carried out with all three alternatives to investigate their effect.

Raising the spot temperature by $200 \mathrm{~K}$ or lowering the facular temperature by $150 \mathrm{~K}$ has no recognizable effect on the reconstruction. When both effects are applied, reconstruction near the equator is unsatisfactory. The southernmost part of the spot is missing, and there is a rather wide gap between the spot and the ring to the south. The southernmost part of the ring is incorrectly shifted towards the equator and is too hot, which again illustrates the problems with latitude resolution near the equator.

When the ring is made $200 \mathrm{~K}$ hotter than the photosphere, it is still recovered, but faint. The ring is clearly hotter than the high-temperature part of the noise patterns on the stellar surface outside the active region (see Fig. 3). The ring disappears when its temperature is just $100 \mathrm{~K}$ above the photospheric temperature, and it gets lost in the temperature noise on the surface. 
This shows that faculae with a temperature contrast like solar faculae are not detectable with Doppler imaging, even if they have a larger area than solar faculae.

\subsubsection{Varying the stellar inclination}

Inclination $i$ of the stellar rotational axis was $i=60^{\circ}$ in the original inversion in Fig. 3. $i$ should ideally be between $30^{\circ}$ and $60^{\circ}$ for Doppler imaging to produce a reliable result (Vogt et al. 1987). If the inclination is higher, the star is viewed almost equator-on, and the inversion algorithm cannot distinguish between the northern and southern hemispheres. Lower inclination implies viewing the star almost pole-on, and the rotational broadening of the spectral line profiles is severely reduced. Since $i=60^{\circ}$ is already quite high, only a test with lower inclination $\left(i=30^{\circ}\right)$ was carried out.

The facular ring is in this case recovered, although its southern part has a somewhat lower temperature than in the input. This is because the southern part of the ring is always close to the limb whenever it is visible, and thus contributes little to the integrated line profile.

\subsubsection{Polar spot}

Polar spots have been found in most of the RS CVn-type stars that have been targets for Doppler imaging (Strassmeier 2002). To determine whether the presence of a polar spot influences the reconstruction of the facular ring, a test was made where the stellar surface also contained a polar spot with the same temperature as the spot surrounded by a facular ring. The polar spot reached down to latitude $63^{\circ}$.

The spot, the ring, and the polar spot are all recovered, but their temperatures, sizes, and positions are not all correct. The southern part of the ring is wider than in the input, and there is a weak ghost spot south of the equator, illustrating the reduced latitude resolution near the equator. The region of the polar spot that lies closest to the rotational pole ends up with photospheric temperature in the reconstruction. Surface elements in this region have a small area and small Doppler shifts due to rotation, which means that changing the temperature in them will have a negligible effect on the integrated line profile.

\subsubsection{More than one active region}

To test whether the Doppler imaging technique is able to reconstruct more than one active region like the one in Fig. 1, tests were made where the surface contained two and three such active regions. The regions were all at the same latitude and had a maximum separation in longitude. In both cases all the active regions are satisfactorily recovered.

\subsection{A more realistic example: UX Ari}

We saw in Sect. 3.2 how three different parameters of the spectroscopic observations and of the target star affect the surface resolution in the resulting Doppler imaging map. The parameters were, however, varied one by one. In a realistic case, both the signal-to-noise ratio and the rotational phase coverage of the spectroscopic observations are likely to be poorer than in the ideal case (Fig. 3). Further, in the case of our target star UX Ari (Aarum-Ulvås et al., in prep.), the projected equatorial rotational velocity is about half of what it was in Fig. 3.

To illustrate more clearly what would be the outcome in a case closer to reality, synthetic observations were generated with $S / N=200$ at 20 rotational phases for a star with $v \sin i=40 \mathrm{~km} \mathrm{~s}^{-1}$. The synthetic spectroscopic observations were subsequently inverted using the Fe I $6141.7 \AA$ and Si I $6155.2 \AA$ lines and 40 iterations, and the result is presented in Fig. 4. Again, the inversions began with the presumption of a smooth stellar surface. In this case the ring is not really recognizable as a ring; although there are facular areas to the north, west, and south of the spot, they are not connected. The facular area north of the spot is small, and its temperature is lower than the input facular temperature. The facular area to the west of the spot is incorrectly extended and widened towards the equator, while the facular area south of the spot is wider than the input ring. There is an isolated facular area crossing the central meridian between phases 0.60 and 0.65 around latitude $30^{\circ}$ that is not present in the input surface. Finally, there is a slightly cooler area than the photosphere on the equator just south of the active region.

The mean deviation between the "observed" spectral line profiles and the model spectral line profiles in Fig. 4 is $0.481 \%$, corresponding to $S / N=208$ in the synthetic observations. The reconstructed spot and facular temperatures (3754 K and $5196 \mathrm{~K}$, respectively) are approximately as in the previous case, as is the behaviour of the reconstructed photometry (Fig. 3).

This inversion demonstrates that in the case of UX Ari with real spectroscopic observations, it would not be possible to completely recover a facular ring like the one in Fig. 1. Parts of the ring are recovered, but some of the parts have incorrect sizes (both too small and too large), and there are also facular areas in the resulting stellar surface map that are not present in the input surface. This calls for caution when interpreting facular areas in Doppler imaging maps derived from real spectroscopic observations.

Realistic spectroscopic observations of UX Ari, or any other target star, are likely to have two additional properties that have not been investigated here. The observations are not likely to be equidistant in rotational phase, and they are not all likely to have the same signal-to-noise ratio, which will both obviously affect the surface resolution in the resulting map. The expected effect of the former is to improve the resolution wherever the observations are less than 0.05 apart in rotational phase, as they were in Fig. 4. Correspondingly, the resolution should be reduced where the observations are farther apart in phase.

\section{Summary and conclusions}

The main issue addressed in this paper is the ability of the Doppler imaging technique to reconstruct stellar-surface active regions where the brightness varies strongly along lines of constant radial velocity. These lines are chords across the stellar surface parallel to the stellar rotational axis 

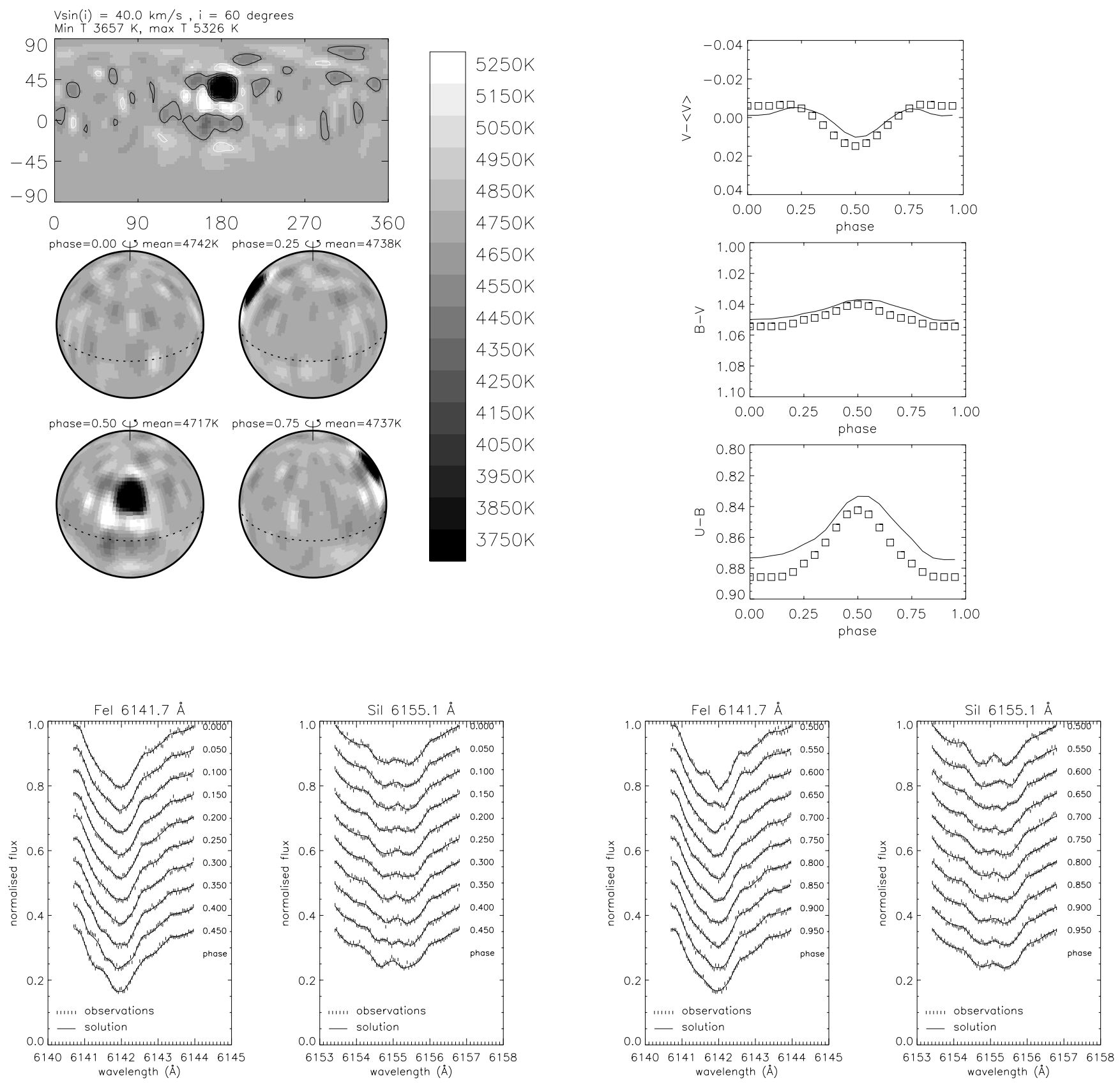

Fig. 4. The same as Fig. 3, but for more realistic observations.

(Vogt \& Penrod 1983). The rotation of the star makes it possible to resolve the brightness variations along these chords, but it requires sufficient phase coverage and signal-to-noise ratio in the observed spectra.

Careful choice of spectral lines in the Doppler imaging process will improve the reconstruction. A set of spectral lines that react differently to a given temperature variation makes it possible to trace the flux variations in the observed spectral line profiles back to temperature variations on the stellar surface in a more consistent and unambiguous way.

The inversions presented here have shown that the Doppler imaging technique is able to reconstruct stellar-surface active regions with high temperature contrast along lines of constant radial velocity. However, a successful reconstruction requires more iterations than is common and spectroscopic observations with a very dense rotational phase coverage, 40 in this case. A large number of iterations is only helpful when the observations have a high signal-to-noise ratio, 400 in this paper.

The RS CVn system UX Ari was used in this paper as an example of a realistic case. For this star the facular ring was broken up in parts, and not all the parts came out with their correct widths. Also, some false facular areas were seen in the resulting stellar surface map. It appears that in realistic cases, the reconstructed map will show a spot surrounded by facular areas that are not connected. Furthermore, the facular areas will tend to be more pronounced towards the stellar equator. If such 
features show up in Doppler imaging maps derived from real observations, it could suggest that the spot is surrounded by a facular ring.

Acknowledgements. The author would like to thank Oddbjørn Engvold and Heidi Korhonen for their careful reading of the manuscript and many useful comments. Thomas Hackman has contributed with many interesting and useful discussions of the computer codes for generating synthetic data and spectral line profile inversions. Thanks also go to the referee and the A\&A Language Editor, whose comments and remarks have improved the paper further. Parts of this work have been supported by the German Research Foundation (DFG) under project number TW9249-DFG STR 645/1-2.

\section{References}

Aarum Ulvås, V., \& Engvold, O. 2003, A\&A, 399, L11

Aarum Ulvås, V., \& Henry, G. W. 2003, A\&A, 402, 1033

Donati, J.-F., Brown, S. F., Semel, M., et al. 1992, A\&A, 265, 682

Foukal, P. V. 2004, Solar Astrophys., 2nd ed. (Wiley-VCH), Chap. 8

Hackman, T., Jetsu, L., \& Tuominen, I. 2001, A\&A, 374, 171

Huenemoerder, D. P., Buzasi, D. L., \& Ramsey, L. W. 1989, AJ, 98, 1398
Krivova, N. A., Solanki, S. K., Fligge, M., \& Unruh, Y. C. 2003, A\&A, 399, L1

Lanza, A. F., Rodonò, M., Mazzola, L., \& Messina, S. 2001, A\&A, 376,1011

Piskunov, N. E. 1991, in The Sun and Cool Stars: activity, magnetism, dynamos, ed. I. Tuominen, D. Moss, \& G. Rüdiger (Springer-Verlag), IAU Coll., 130, 309

Piskunov, N. E., Tuominen, I., \& Vilhu, O. 1990, A\&A, 230, 363

Rast, M. P., Meisner, R. W., Lites, B. W., Fox, P. A., \& White, O. R. 2001, ApJ, 557, 864

Rice, J. B., Wehlau, W. H., \& Khokhlova, V. L. 1989, A\&A, 208, 179

Saar, S. H., Piskunov, N. E., \& Tuominen, I. 1992, in PASPC, Vol. 26, Cool Stars, Stellar Systems, and the Sun, Seventh Cambridge Workshop, ed. M. S. Giampapa, \& J. A. Bookbinder, 255

Strassmeier, K. G. 1994, A\&A, 281, 395

Strassmeier, K. G. 2002, AN, 323, 309

Strassmeier, K. G., Rice, J. B., Wehlau, W. H., Hill, G. M., \& Matthews, J. M. 1993, A\&A, 268, 671

Taş, G., Evren, S., \& İbanoğlu, C. 1999, A\&A, 349, 546

Vogt, S. S., \& Hatzes, A. P. 1991, in The Sun and Cool Stars: activity, magnetism, dynamos, ed. I. Tuominen, D. Moss, \& G. Rüdiger (Springer-Verlag), IAU Coll., 130, 297

Vogt, S. S., \& Penrod, G. D. 1983, PASP, 95, 565

Vogt, S. S., Penrod, G. D., \& Hatzes, A. P. 1987, ApJ, 321, 496 